Check for updates

Cite this: J. Mater. Chem. C, 2018, 6, 781

Received 18th October 2017 , Accepted 11th December 2017

DOI: $10.1039 / \mathrm{c} 7 \mathrm{tc0} 4757 f$

rsc.li/materials-c

\section{Spectroscopic characterization of the structural properties of quinoxalinophenanthrophenazine thin films $\uparrow$}

\author{
Ewelina Z. Fratczak, ${ }^{a}$ Tomasz Makowski, (D) ${ }^{\mathrm{b}}$ Rasha M. Moustafa, ${ }^{\mathrm{c}}$ Tarek H. El-Assaad, \\ Marek E. Moneta, ${ }^{a}$ Pawel Uznanski ${ }^{\star b}$ and Bilal R. Kaafarani (D) *c
}

\begin{abstract}
Thin films of 2,11-bis(1,1-dimethylethyl)-6,7,15,16-tetramethylquinoxalino $\left[2^{\prime}, 3^{\prime}: 9,10\right]$ phenanthro[4,5abc]phenazine (TQPP-Me) and its long chain alkoxy derivative 2,11-bis(1,1-dimethylethyl)-6,7,15,16tetrakis(dodecyloxy)quinoxalino[2', $\left.3^{\prime}: 9,10\right]$ phenanthro[4,5-abc]phenazine (TQPP-OC 12 ) on silicon/native silica $\left(\mathrm{Si} / \mathrm{SiO}_{2}\right)_{\text {native }}$ and fused silica substrates were analyzed in respect of their microstructural and anisotropic optical properties. The molecules, considered as candidates for organic electronic applications, have different solubilities, hence the fabrication of their solid layers was carried out by various methods. Anisotropy resulting from the alignment and shape of the organic molecules was induced by the choice of sedimentation method on inorganic substrates. TQPP-Me thin films were obtained by organic molecular beam deposition in ultra-high vacuum conditions while TQPP-OC 12 layers were produced from solution by spin coating. The alignment and microstructure of TQPP-Me and TQPP-OC 12 molecules in the formed films were studied by optical microscopy, atomic force microscopy, polarized infrared measurements in transmission and reflection modes, polarized UV-Vis absorption and fluorescence spectroscopy, and spectroscopic ellipsometry. Optical properties and morphology of both studied films exhibit different textures with strongly uniaxial anisotropy in both cases. The TQPP-Me vacuum-deposited molecules are arranged parallel to the surface while the cores of spin-coated TQPP-OC 12 are cofacially packed and tilted with respect to the surface normal. Ellipsometric measurements were fitted with an isotropic model; however, the results do not exclude uniaxial ordering of the investigated samples.
\end{abstract}

\section{Introduction}

The structural organization of organic compounds greatly influences the functioning, performance and lifetime of modern electronic devices, such as organic light emitting diodes (OLEDs), organic field effect transistors (OFETs) or organic photovoltaic cells (OPVs). ${ }^{1,2}$ Highly ordered active organic layers can be produced, for example, on functionalized inorganic substrates as ordered interface structures between the active layer and dielectrics. ${ }^{3}$ It is well recognized that the molecular organization determines the morphological properties, which strongly affect photo- and electro-optical, and charge transport properties.

\footnotetext{
${ }^{a}$ Faculty of Physics and Applied Informatics, University of Lodz, Pomorska 149/153, 90-236 Lodz, Poland

${ }^{b}$ Centre of Molecular and Macromolecular Studies, Polish Academy of Sciences, Sienkiewicza 112, 90-363, Lodz, Poland. E-mail: puznansk@cbmm.lodz.pl ${ }^{c}$ Department of Chemistry, American University of Beirut, Beirut 1107-2020, Lebanon.E-mail: bilal.kaafarani@aub.edu.lb

$\dagger$ Electronic supplementary information (ESI) available: Optical microscopy images for TQPP-OC ${ }_{12}$ and TQPP-Me under polarized and cross polarized light. See DOI: $10.1039 / \mathrm{c} 7 \mathrm{tc} 04757 \mathrm{f}$
}

Organic molecules deposited on modified inorganic surfaces show various molecular orientations and anisotropic properties, depending on the structure of the molecule and the nature of the substrate. Nowadays, the most important processing strategies for organic thin film growth are based on vacuum deposition, on the use of surfactants or self-assembled monolayers, ${ }^{3-8}$ or on modern low-cost processing, such as aerosol-jet ${ }^{9}$ and inkjet printing, ${ }^{10}$ screen printing, ${ }^{11}$ spin $^{12}$ or spray coating, ${ }^{13}$ and zone casting. ${ }^{14}$ The organic molecular beam deposition method is very effective for small molecules such as pentacene $e^{7,15-21}$ or rubrene $e^{7,22}$ over rather small areas. ${ }^{23}$ On the other hand, solution casting techniques are used to align structurally complicated materials which are difficult to assemble by other methods over larger areas. ${ }^{24,25}$ However, there are compounds that can be efficiently processed by both methods in order to use them in organic electronics. ${ }^{26-29}$

Semiconducting heteroaromatic quinoxalinophenanthrophenazine (TQPP) derivatives were developed as potential organic p-type semiconductors. ${ }^{30-37}$ At the same time, however, it turned out that these molecules exhibit interesting solution and solid state fluorescence properties, which depend on their alignment. It has been 
shown that the thin film microstructure of molecules with long aliphatic chains is induced by the choice of sedimentation method on inorganic substrates. ${ }^{25,30}$ Continuous thin films of 2,11-bis(1,1-dimethylethyl)-6,7,15,16-tetrakis(dodecyloxy)quinoxalino$\left[2^{\prime}, 3^{\prime}: 9,10\right]$ phenanthro $[4,5-a b c]$ phenazine $\left(\right.$ TQPP-OC $\left._{12}\right)$ with a thickness of 200-300 nm prepared by a zone-casting method form lamellas arranged parallel to the surface, wherein molecular cores orient almost perpendicularly $\left(\sim 96^{\circ}\right)$ to the substrate. The molecular stacking axis is parallel to the zone-casting direction, with molecules tilted by $\sim 57^{\circ}$. The increased distance between aromatic cores parallel to the casting direction $(6.9 \AA)$ was ascribed to the presence of two tert-butyl substituents at the core of TQPP-OC 12 . The films form large area uniform layers regularly interrupted by long crystalline folds with a thickness of approx. $1 \mu \mathrm{m}$, a height of $85 \mathrm{~nm}$, and with the distance between them in the range of $10-15 \mu \mathrm{m} .^{25}$ Due to their relatively large thickness and complex surface morphology, zone-cast layers may be of little use in organic electronics applications. Therefore, it seems that for further thin film studies, TQPP-OC 12 molecules should be processed by other methods from solution, especially by spin coating which gives layers of controlled and uniform thickness.

To investigate the structure-property relationships of TQPP moieties, TQPP derived polymers were recently synthesized and showed remarkable high porosity and photoluminescence. ${ }^{33,38}$ However, there is still room for research into low molecular weight systems. In this work, we present for the first time a comparative study of structurally anisotropic thin films from 2,11-bis(1,1dimethylethyl)-6,7,15,16-tetramethylquinoxalino[2' $\left[3^{\prime}: 9,10\right]$ phenantro[4,5- $a b c]$ phenazine (TQPP-Me) and its long chain analogue 2,11-bis(1,1-dimethylethyl)-6,7,15,16-tetrakis(dodecyloxy)quinoxalino$\left[2^{\prime}, 3^{\prime}: 9,10\right]$ phenanthro[4,5-abc]phenazine (TQPP-OC 12 ) (Fig. 1), prepared by two complementary deposition methods: organic molecular beam deposition (MBD) in a high vacuum and processing from solution by spin coating (SC). These techniques allow us to obtain uniform thin films of similar microstructure from molecules which are hardly processable from a solution and those which have excellent solubility, respectively. One of the purposes of our study was to demonstrate how a molecular structure modified by the presence of aliphatic substituents can affect the morphology and molecular ordering of thin TQPP-Me and TQPP-OC 12 films. Differences in the chemical structure and deposition method of the studied samples can result in various polymorphs and have an impact on their solid state optical and spectroscopic properties.

The organization of the molecules in thin films was examined by an extremely sensitive infrared (IR) technique - grazing attenuated total reflection - spectroscopic ellipsometry (SE), UV-Vis absorption and fluorescence spectroscopy, and atomic force microscopy (AFM). These combined techniques allow for the independent and complementary determination under ambient conditions of the alignment, anisotropic optical properties and morphology of the deposited layers.

The orientation of the molecules was deduced from vibrational analysis obtained by Fourier transform infrared spectroscopy (FTIR) with an attenuated total reflectance geometry at grazing angle (GATR) and in a transmission mode. The detailed results of the IR studies were completed by UV-Vis and variable angle spectroscopic ellipsometry (VASE) measurements, which can also probe the mean orientation of molecules in thin films. The surface morphologies were examined by AFM. Polarized vibrational modes studied in this work can help to specify the orientation of the samples which, in turn, is responsible for the structural properties and consequently for the charge transport properties. $^{39,40}$

\section{Experimental methods}

2,11-Bis(1,1-dimethylethyl)-6,7,15,16-tetramethylquinoxalino$\left[2^{\prime}, 3^{\prime}: 9,10\right]$ phenantro[4,5-abc]phenazine (TQPP-Me) and 2,11bis(1,1-dimethylethyl)-6,7,15,16-tetrakis(dodecyloxy)quinoxalino $\left[2^{\prime}, 3^{\prime}: 9,10\right]$ phenanthro[4,5- $\left.a b c\right]$ phenazine (TQPP-OC 12 ) (Fig. 1) were synthesized as described earlier ${ }^{34,41}$ and used for thin film preparation. Thin films of TQPP-Me and TQPP-OC 12 were deposited on native $\mathrm{Si} / \mathrm{SiO}_{2}$ substrates or quartz plates. The substrates were cleaned in acetone and alcohol in three $15 \mathrm{~min}$. cycles using an ultrasonic bath, and dried in a nitrogen stream. TQPP-OC $_{12}$ cannot be deposited by vacuum sublimation but its simpler model form, where the alkoxy $-\mathrm{OC}_{12} \mathrm{H}_{25}$ groups are substituted by methyl (-Me) groups can be effortlessly evaporated. Therefore, a thin film of TQPP-Me was deposited by MBD, whereas thin films of TQPP-OC 12 were solution cast under ambient conditions. The growth of TQPP-Me layers was carried out in a high-vacuum chamber at a working pressure of $2 \times 10^{-7} \mathrm{mbar}$.

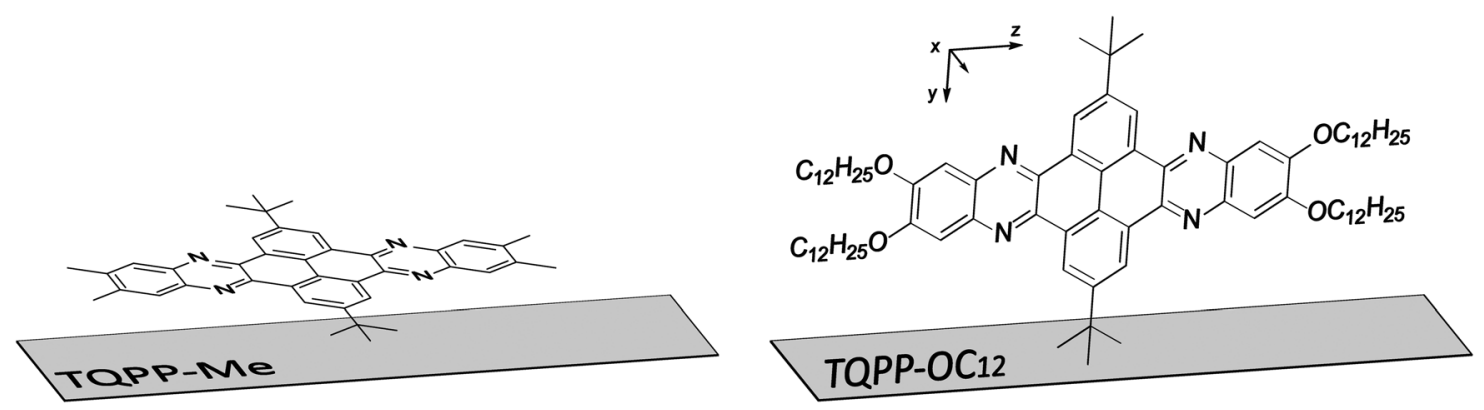

Fig. 1 Chemical structures of TQPP-Me and TQPP-OC 12 and their proposed molecular orientation on the $\mathrm{Si} / \mathrm{SiO}_{2}$ substrate deduced on the basis of this study. The core belongs to $D_{2 h}$ symmetry and all the transition moments lie along one of the three molecular axes $x, y$ or $z$. 
The chamber was equipped with a home-made evaporator located at the bottom, and the sample was placed centrally above the evaporator in the upper part of the chamber. The distance between the source compound and the substrate was $12 \mathrm{~cm}$. TQPP-Me was deposited on the substrate at a temperature of $200{ }^{\circ} \mathrm{C}$ with a deposition rate of $6 \mathrm{~nm} \mathrm{~min}^{-1}$. The thickness growth was monitored during deposition by a quartz crystal microbalance and further confirmed by ellipsometric measurements.

Thin films of TQPP-OC 12 were produced by spin coating (SC) from a filtered solution ( $0.2 \mu \mathrm{m}$ PTFE membrane) in toluene at a concentration of $2 \mathrm{mg} \mathrm{mL} \mathrm{m}^{-1}$ at $1000 \mathrm{rpm}$ onto Si substrates with a natively oxidized layer. The thicknesses of the SC samples were checked by ellipsometric measurements.

Linear dichroism FTIR studies for symmetry assignment of vibrational transitions of TQPP-OC Th $_{12}$ molecules were conducted in an oriented low-density polyethylene (PE). The molecules were introduced into the dissolved polymer in hot toluene. After solvent removal, PE films of $0.75 \mathrm{~mm}$ in thickness were prepared by melting the composite at $165{ }^{\circ} \mathrm{C}$, compressing and fast cooling to $0{ }^{\circ} \mathrm{C}$ in water to diminish the size of the fine spherulitic structure of the semicrystalline PE matrix. Sheet stretching was done on a stretcher with a drawing ratio of $600 \%$. Separate polarized spectra of TQPP-OC 12 were recorded with the electric vector of the analyzing light along the stretching direction $\left(A_{Z}\right)$ and with the electric vector perpendicular to the sample axis $\left(A_{Y}\right)$. The reference (base-line) spectra were obtained from an undoped PE sheet of the same shape and thickness. TQPP-OC $_{12}$ concentration was in the range of 0.1-0.2 absorbance units. A similar TQPP-Me sample could not be prepared because of its low solubility in toluene and PE.

Vibrational spectra were measured by IR spectroscopy on a Thermo Nicolet 6700 FTIR spectrometer with $4 \mathrm{~cm}^{-1}$ resolution. Attenuated total reflection spectra (ATR) were measured using a single reflection Golden Gate accessory (Specac) with a Ge-ATR crystal at an incident angle of $45^{\circ}$. Grazing angle attenuated reflection spectra (GATR) were measured using the VariGATR accessory (Harrick Sci. Prod.). Both unpolarized, p-polarized (electric field vector in the plane of incidence) and s-polarized (electric field vector perpendicular to the plane of incidence) IR spectra at $63^{\circ}$ incidence optimized for the highest sensitivity were recorded using a KRS-5 gold wire grid polarizer.

Optical parameters were determined by means of spectroscopic ellipsometry using J. A. Woollam V-VASE apparatus at three different angles of light incidence from $60^{\circ}$ to $70^{\circ}$ in steps of $5^{\circ}$. Experimental ellipsometric parameters $\Psi(\lambda)$ and $\Delta(\lambda)$ were collected in steps of $2 \mathrm{~nm}$ throughout the spectral range from 260 to $1000 \mathrm{~nm}$. The ellipsometry data were analyzed using commercial WVASE32 software. Modeling of experimental data and determination of optical properties, and the film thickness were carried out by application of a uniaxial and isotropic model of dielectric function.

UV-Vis absorption and emission measurements were performed using an HP 8453 diode array spectrophotometer and Fluorolog-3 22 instrument (Horiba Jobin-Yvone). Thin film fluorescence spectra were performed with a solid sample holder tilted at $30^{\circ}$ to the excitation light. The quantum yield was measured using a Quanta-phi integrating sphere (Horiba). Fluorescence lifetimes were obtained using a time-correlated single-photon counting (TCSPS) accessory (Horiba) and fluorescence decay analysis software (DAS6). The samples were excited with a $374 \mathrm{~nm}$ laser diode pulsed at $1 \mathrm{MHz}$ and the decay curves were recorded at a wavelength of $425 \mathrm{~nm}$ (for TQPP-Me) or $435 \mathrm{~nm}$ (for TQPP-OC 12 ) using TCSPC. The prompt-deconvoluted fit of the fluorescence decay curves yielded a monoexponential behavior.

The surface topography and morphology of the deposited layers were measured by an AFM microscope set in tapping mode in a height contrast under ambient atmosphere at $25{ }^{\circ} \mathrm{C}$. The instrument was a Nanosurf Flex Axiom with a C3000 controller (Nanosurf AG, Switzerland) equipped with a commercially available rectangular probe (PPP-NCHR Nanosensors). AFM images were recorded with an available scanner sampling resolution of $512 \times 512$ data points. Image analysis was performed using SPIP Image Metrology software (Denmark).

$\mathrm{X}$-ray diffraction patterns of thin film samples were collected using a Panalytical X'PERT MPD diffractometer for a $2 \theta$ range of $3^{\circ}$ to $50^{\circ}$ at an angular resolution of $0.1^{\circ}$ working in the grazing incidence mode with Co-K $\alpha$ radiation (1.7890 ̊).

\section{Results and discussion}

One of the purposes of our study was to demonstrate how a molecular structure modified by the presence of aliphatic substituents can affect the morphology and molecular ordering of thin TQPP-Me and TQPP-OC 12 films. Differences in chemical structure and deposition method of the studied samples can result in various polymorphs and have an impact on their solid state optical and spectroscopic properties.

\section{Grazing incidence XRD, optical microscopy and AFM examinations}

Thin films of TQPP-OC 12 were deposited on Si substrate by spin coating which gives layers of uniform thickness. The thickness of such films can be easily controlled, without changing the morphology that resembles tangled fibers (Fig. 2a).

Films deposited in high vacuum from TQPP-Me (Fig. 2b) demonstrate tiny and elongated grains with a more packed morphology than the spin-coated TQPP-OC 12 sample. These crystalline structures with a baton-like shape are distributed randomly (the TQPP-OC 12 structures are organized more directionally). Both films are uniform, as is evident from optical microscope examinations taken in crossed polarizers, where total image extinction was observed independent of azimuthal direction, indicating uniaxial alignment.

The crystal phase composition of the samples was measured with grazing incidence X-ray diffraction (GIXRD). Fig. 3 shows the reflection patterns for as-obtained spin-coated TQPP-OC 12 and molecularly deposited TQPP-Me thin films. X-ray diffractograms demonstrate a progression of fast-fading reflection peaks of low intensity, which can be interpreted in terms of 

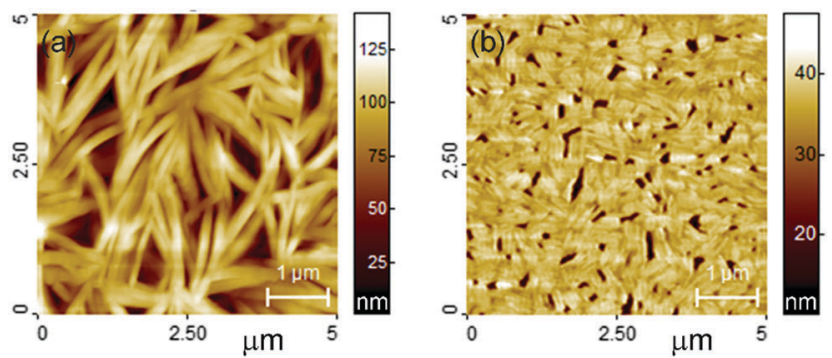

Fig. 2 AFM topography images of thin films of (a) spin-coated TQPP$\mathrm{OC}_{12}$ and (b) deposited by MBD TQPP-Me on $\left(\mathrm{Si} / \mathrm{SiO}_{2}\right)_{\text {native }}$ substrate. TQPP- $\mathrm{OC}_{12}$ creates crystallites in the form of tangled dry grass. For TQPP-Me well-packed, regular crystalline grains of a medium length of about $300 \mathrm{~nm}$, a width of about $50 \mathrm{~nm}$, and a diameter of about $3 \mathrm{~nm}$ are well visible. The calculated RMS roughness of the surface is $1.9 \mathrm{~nm}$.

oriented molecular structures. The positions of the observed diffraction patterns of TQPP-OC 12 suggest the coexistence of two polymorphic phases. The peaks corresponding to the $d$-spacing of 19.9 and $9.9 \AA$ belong to the same family and are characteristic for a phase of as-cast (non-annealed) films. ${ }^{25}$ The peak at $14.1 \AA$ is assigned to the surface effects and disarrangement of the crystal lattice. The diffractogram for TQPP-Me can be interpreted in a similar way, where the $d$-spacing forms a less correlated family of reflections. This indicates the wealth of disorder effects toward the surface for a crystal lattice grown from molecularly deposited films at high temperature.

\section{Infrared spectroscopy of TQPP thin films}

The molecular alignment of TQPP-Me and TQPP-OC ${ }_{12}$ in thin films can be determined by polarized IR spectroscopy using the grazing angle GATR technique, in which a thin organic layer is sandwiched between Ge-ATR crystal and the Si substrate, and the analyzing wave propagates parallel to the film/silicon interface. In GATR geometry, the electric field strength perpendicular to the Ge surface is enhanced within the film. ${ }^{42}$ Thus, using

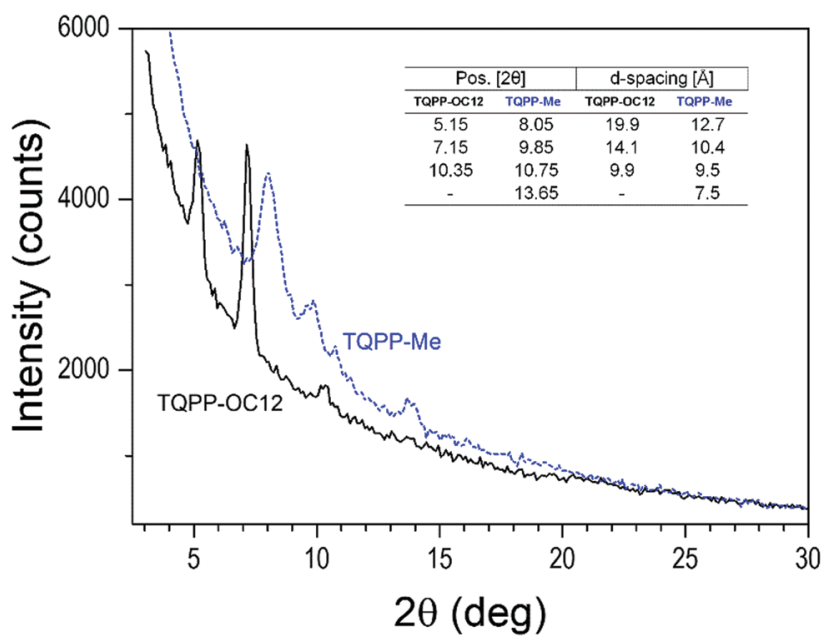

Fig. 3 GIXRD pattern of the TQPP-OC 12 spin-coated and vacuumdeposited TQPP-Me layers. The $2 \theta$ positions and the $d$-spacing calculated from Bragg's law are listed in the inset. p- and s-polarized IR radiation, one can observe components of transition dipole moments parallel $(\mathrm{p}$ ) or perpendicular (s) to the plane of IR light incidence. However, analysis of molecular ordering in thin films with spectroscopic methods requires knowledge of the polarization of each transition moment within the molecular framework.

Both quinoxalinophenanthrophenazine molecules belong to $D_{2 \mathrm{~h}}$ symmetry and infrared active vibrational modes are polarized along the three molecular axes $z, y$ and $x$, defined in Fig. 1. The assignment of the most intensive IR transitions to their symmetries for the core of similar structure were based on quantum chemical calculations and have been reported already. ${ }^{30}$ In the case of the examined molecules with tertbutyl substituents, the directional properties of spectroscopic transitions can be experimentally determined in a fairly easy way: namely, symmetry assignments of vibrations can be conducted from dichroic IR spectra of the solute uniaxially oriented in a stretched polymer matrix. ${ }^{43}$ Fig. 4 shows polarized IR spectra of TQPP-OC 12 in a stretched polyethylene matrix parallel $\left(A_{Z}\right)$ and perpendicular $\left(A_{Y}\right)$ to the stretching direction. Some bands in the ranges 700-750, 1455-1485, and $2800-3000 \mathrm{~cm}^{-1}$ are obscured by the absorption of the polymer matrix. Non-overlapping bands characterized by the dichroic ratio, $d=A_{Z} / A_{Y}$, can be arranged into three groups with values $d=5.5,0.52$, and 0.17 . The dichroic ratios are related to the average degree of alignment of the three molecular axes $u=z, y$, and $x$ by the relationship $\left\langle\cos ^{2} u\right\rangle=d_{u} /\left(d_{u}+2\right)$, where $\operatorname{arc}(\cos u)$ is the angle between the $u$-th axis and the polymer stretching direction $Z$, and $d_{u}$ is the observed dichroic ratio for transitions polarized along the $u$-th axis. The three orientation factors $\left\langle\cos ^{2} u\right\rangle=0.72,0.2$, and 0.08 sum to unity and determined polarization of allowed IR vibrations, which lie along one of the three symmetry axes $z, y$, and $x$. The symmetry assignment of

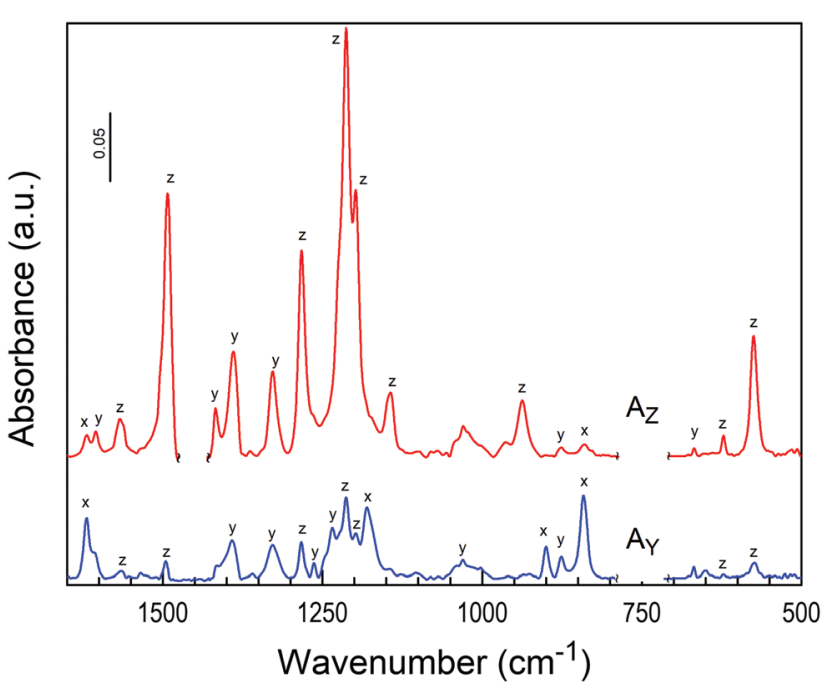

Fig. 4 Polarized infrared spectra of TQPP-OC 12 in stretched polyethylene: parallel $\left(A_{Z}\right)$ and perpendicular $\left(A_{Y}\right)$ to the stretching direction. Assigned polarizations are labelled according to molecular axes: $z$ is the long in-plane axis, $y$ is the short in-plane axis, and $x$ is the out-of-plane axis (see definition in Fig. 1). 


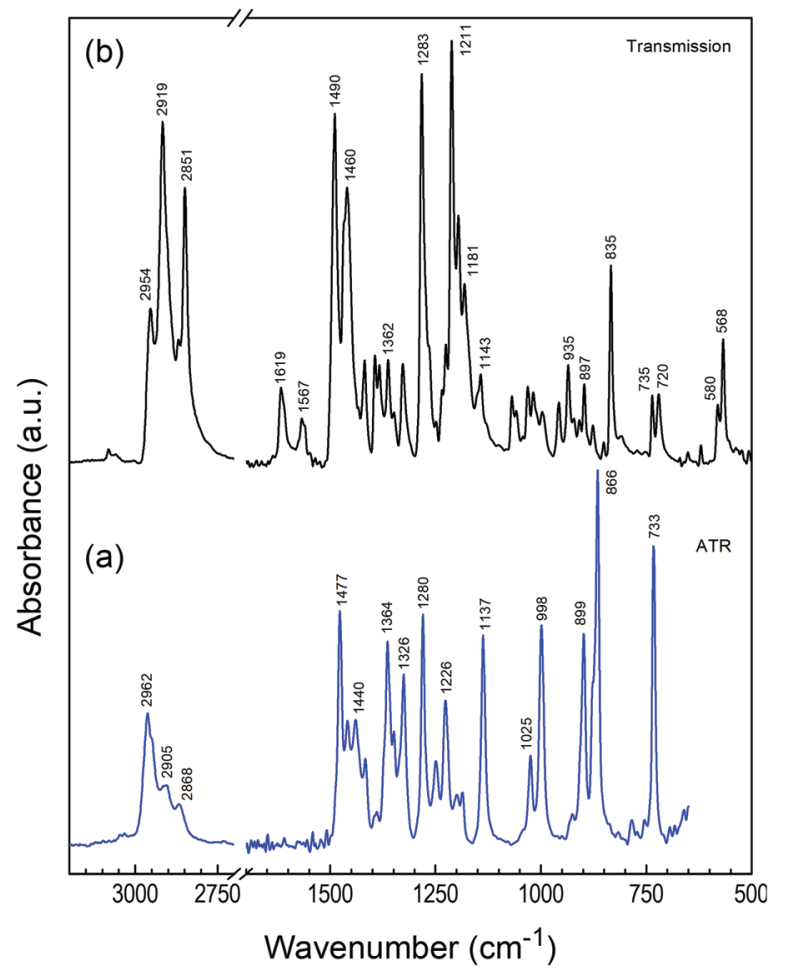

Fig. 5 FTIR spectra of polycrystalline TQPP-Me (a) and TQPP-OC $C_{12}$ (b).

some bands for the TQPP-OC 12 molecule is given in Fig. 4 and can be compared with its microcrystalline powder sample spectrum detected in transmission mode (Fig. 5b), which is assumed to be composed of randomly oriented microcrystals. The positions of the most intense IR peaks with their assignment to defined vibrational modes are marked in Fig. 5 and collected in Table 1. According to the assignment, most transitions lie in the molecular plane of TQPP-OC ${ }_{12}$, except for those at 1619, 1181, 897 and $835 \mathrm{~cm}^{-1}$, which show the out-of-plane polarization. The IR spectra of both compounds are similar, although they vary in the intensity of individual bands (Fig. 5).

Fig. 6 shows GATR spectra measured with p- and s-polarized light for TQPP-Me and TQPP-OC ${ }_{12}$ thin films deposited in a high vacuum and from solution by spin coating, respectively. Comparing p- and s-polarized spectra and the associated ratio of peak intensities, $A_{\mathrm{p}} / A_{\mathrm{s}}$, for TQPP-Me, it can be seen that most of the peaks of the s-polarized spectrum are intensified prior to the p-polarized spectrum. Only in the range of $1620-1220 \mathrm{~cm}^{-1}$ are the following maxima of s-spectrum attenuated: 1610, 1416, 1251 and $1225 \mathrm{~cm}^{-1}$ (Fig. 6a and Table 1). For the TQPP-OC 12 sample, the s-polarized IR signal is much weaker than the p-polarized one over the whole range. The maxima of s-polarized spectrum gain in intensity, as reflected in the $A_{\mathrm{p}} / A_{\mathrm{s}}$ values (Fig. $6 \mathrm{~b}$ and Table 1 ). Taking into account that the IR spectra of both compounds are similar, although they vary in the intensity of individual bands, the observed inverse in intensity between p- and s-polarized spectra suggests that the TQPP molecules are arranged with their cores in a different way relative to the surface of the $\left(\mathrm{Si} / \mathrm{SiO}_{2}\right)_{\text {native }}$ substrate.

Based on the significant intensity enhancement of the s-polarized spectrum for TQPP-Me, one can assume that its
Table 1 Observed vibrations of TQPP-Me and TQPP-OC 12 polycrystalline powders, polarization assignments (for TQPP- $\mathrm{OC}_{12}$ ) and relative intensities $A_{\mathrm{p}} / A_{\mathrm{s}}$ of the IR bands for their thin films on $\mathrm{Si} / \mathrm{SiO}_{2}$ substrates measured in polarized GATR configuration geometry

\begin{tabular}{|c|c|c|c|c|c|}
\hline \multirow[b]{2}{*}{ Mode } & \multicolumn{2}{|c|}{ Wavenumber } & \multirow{2}{*}{$\begin{array}{l}\text { Polarization }^{a} \\
\text { TQPP-OC }_{12}\end{array}$} & \multicolumn{2}{|c|}{$\begin{array}{l}\text { Intensity } \\
\text { ratio } A_{\mathrm{p}} / A_{\mathrm{s}}{ }^{b} \\
\end{array}$} \\
\hline & TQPP-Me & TQPP-OC $_{12}$ & & $\begin{array}{l}\text { TQPP- } \\
\mathrm{Me}\end{array}$ & $\begin{array}{l}\text { TQPP- } \\
\text { OC }_{12}\end{array}$ \\
\hline \multirow{2}{*}{\multicolumn{2}{|c|}{$\begin{array}{l}\text { Core def. } \\
\delta\left(\mathrm{CH}_{2}\right)\end{array}$}} & 568,580 & \multicolumn{3}{|l|}{$z$} \\
\hline & & 720 & & & 5.5 \\
\hline \multirow{2}{*}{$\begin{array}{l}\text { Core def. o.p. } \\
\mathrm{CH} \text { o.p. }\end{array}$} & \multirow[t]{2}{*}{733} & 735 & & \multirow[t]{2}{*}{0.4} & 4.0 \\
\hline & & 835 & $x$ & & 3.5 \\
\hline $\mathrm{CH}$ o.p. & \multicolumn{2}{|l|}{866} & & \multicolumn{2}{|l|}{0.7} \\
\hline $\mathrm{C}-\mathrm{C}$ (t-but.) & \multirow{2}{*}{899} & 897 & $x$ & \multirow{2}{*}{0.3} & 2.6 \\
\hline Core str. & & 935 & $z$ & & 2.0 \\
\hline Core vibr. & 998 & 998 & & 0.4 & 5.0 \\
\hline \multirow{2}{*}{ Core def. } & 1025 & 1019, 1033 & $y$ & \multicolumn{2}{|l|}{0.3} \\
\hline & 1137 & 1143 & $z$ & & 0.8 \\
\hline CH i.p. & 1186 & 1181 & $x$ & 0.4 & 3.0 \\
\hline Core & 1200 & 1196 & $z$ & & 1.0 \\
\hline \multirow[t]{3}{*}{$\mathrm{C}-\mathrm{O}-\mathrm{C}$} & & 1211 & $z$ & & 1.5 \\
\hline & \multirow[t]{2}{*}{1225} & & & \multirow[t]{2}{*}{1.3} & \\
\hline & & 1236 & $y$ & & 3.3 \\
\hline Core sym. vibr. & 1280 & 1283 & $z$ & 0.4 & 1.2 \\
\hline Core & 1326 & 1330 & $y$ & 0.2 & 1.0 \\
\hline$\delta_{\mathrm{s}}\left(\mathrm{CH}_{3}\right)$ & \multirow[t]{2}{*}{1364} & 1362 & & \multirow[t]{2}{*}{0.4} & 1.5 \\
\hline$\delta_{\mathrm{s}}\left(\mathrm{CH}_{3}\right)$ & & 1386 & \multirow[t]{2}{*}{$z$} & & 1.5 \\
\hline Core vibr. & 1417 & 1420 & & \multicolumn{2}{|l|}{$\begin{array}{l}1.8 \\
0.4\end{array}$} \\
\hline CC str. & \multicolumn{2}{|l|}{1440} & & \multicolumn{2}{|l|}{0.4} \\
\hline$\delta_{\mathrm{as}}\left(\mathrm{CH}_{3}\right)$ & \multirow[t]{2}{*}{1459} & 1460 & & \multirow[t]{2}{*}{0.5} & 1.3 \\
\hline$\delta\left(\mathrm{CH}_{2}\right)$ & & 1469 & & & 1.9 \\
\hline Core str. & \multicolumn{2}{|l|}{1477} & & 0.5 & \\
\hline Core str. & & 1490 & $z$ & & 1.3 \\
\hline Core str. & & 1567 & $z$ & & 1.0 \\
\hline Core str. & 1610 & 1605 & $y$ & 5.0 & \\
\hline Core str. & & 1619 & $x$ & & 3.6 \\
\hline$\nu_{\mathrm{s}}\left(\mathrm{CH}_{2}\right)$ & & 2851 & & & 1.7 \\
\hline$\nu_{\mathrm{a}}\left(\mathrm{CH}_{3}\right)$ & 2868 & 2870 & & & \\
\hline$\nu_{\mathrm{a}}\left(\mathrm{CH}_{3}\right)$ & 2905 & & & & \\
\hline$\nu_{\text {as }}\left(\mathrm{CH}_{2}\right)$ & & 2919 & & & 2.3 \\
\hline$\nu_{\text {as }}\left(\mathrm{CH}_{3}\right)$ & 2952 & 2954 & & 3.0 & \\
\hline$\nu_{\text {as }}\left(\mathrm{CH}_{3}\right)$ & 2962 & & & 1.0 & \\
\hline$\nu\left(\mathrm{CH}_{\text {core }}\right)$ & 3031,3045 & 3060,3080 & & & \\
\hline
\end{tabular}

molecular plane is rather parallel to the substrate. The intensity enhancement of the p-polarized spectrum for TQPP-OC 12 is associated with a perpendicular (tilted) orientation of the molecular core relative to the Si plane, whereby it is difficult to indicate the direction of inclination of the molecular $z$ and $y$ axis to the surface. Additionally, a transmission spectrum (not shown) recorded only for TQPP-OC ${ }_{12}$ provides useful information on the molecular orientation on the $\mathrm{Si} / \mathrm{SiO}_{2}$ surface. At normal incidence, an unpolarized IR beam excites only the vibrational modes with transition dipole components parallel to the substrate surface. The transmission TQPP-OC ${ }_{12}$ spectrum overlaps with the s-polarized GATR spectrum, demonstrating the correctness of the GATR measurements.

\section{Photophysical properties}

Detailed photophysical studies of TQPP-OC ${ }_{12}$ in solution and solid state were described earlier ${ }^{25,41,44}$ and no correlation was 


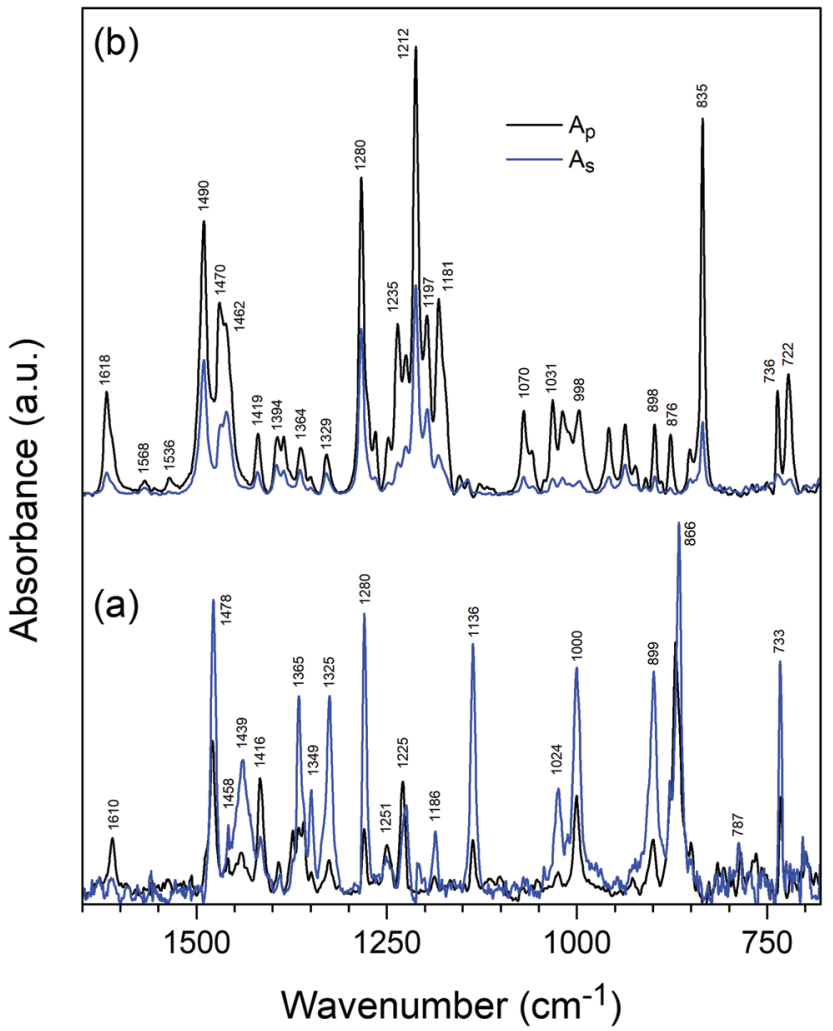

Fig. 6 Polarized GATR infrared spectra of thin films of molecularly deposited TQPP-Me (a) and spin-coated TQPP-OC 12 (b).

found between the chain length of the four alkyl substituents and the Stokes shift, fluorescence quantum yield, fluorescence lifetime, or radiative and nonradiative rate constants. Herein, we reexamine the photophysical properties of TQPP-OC ${ }_{12}$ in an SC thin film and with relation to those observed for TQPP-Me. The short chain analogue of quinoxalinophenanthrophenazine in toluene shows a regular absorption spectrum devoid of vibronic substructure; however, it is hypsochromically shifted by $9 \mathrm{~nm}$ as compared to TQPP-OC 12 (Fig. 7 and 8). Two bands with maxima at $333 \mathrm{~nm}$ and $420 \mathrm{~nm}$ and characteristic of an aromatic compound vibronic progressive structure are observed. The vibronic bands are separated, calculating in the direction of increasing energy, by 1443 and $1344 \mathrm{~cm}^{-1}$, for the low-energy band, and by 1516 and $1349 \mathrm{~cm}^{-1}$, for the second transition band. The emission spectrum shows a maximum at $425 \mathrm{~nm}$, indicating a Stokes shift of $280 \mathrm{~cm}^{-1}$, similar to that for TQPP-OC $12\left(215 \mathrm{~cm}^{-1}\right)$. The fluorescence lifetime $\tau$ in nitrogen-saturated toluene was $\tau=1.06 \mathrm{~ns}\left(\chi^{2}=0.89\right)$ and is shorter than that for TQPP-OC 12 , which was $\tau=1.91 \mathrm{~ns}\left(\chi^{2}=0.96\right)$. Similarly, the value of quantum yield for TQPP-Me $\varphi=10.62 \pm 0.39 \%$ is half that for the long chain analogue $\varphi=21.62 \pm 0.77 \%$.

The absorption spectrum of vacuum-deposited thin film of TQPP-Me shows similar vibronic progressive of the first electronic transition with energy separation of 1362 and $1330 \mathrm{~cm}^{-1}$ coupled to core skeletal vibration and a red-shift from 420 to $432 \mathrm{~nm}$ with respect to the spectrum in toluene (Fig. 7a). Such a shift can be explained by an arrangement of TQPP-Me molecule
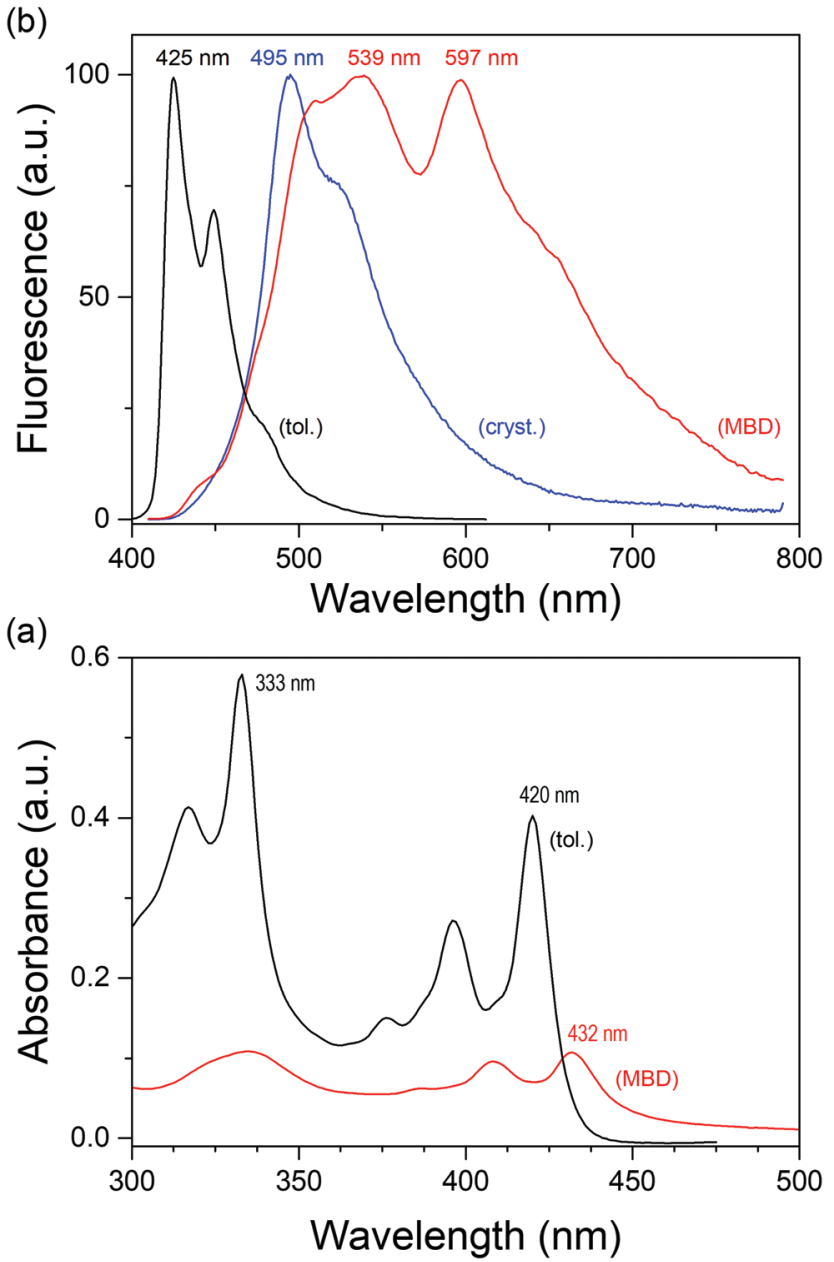

Fig. 7 UV-Vis absorption (a) and fluorescence (b) spectra of TQPP-Me in toluene solution (tol.) and thin film molecularly deposited in high vacuum (MBD) on a quartz substrate. For comparison a fluorescence spectrum of polycrystalline powder (cryst.) is shown.

cores which should be shifted relative to one another. The fluorescence spectrum of the MBD TQPP-Me film shows a more profound red-shift from $425 \mathrm{~nm}$ to $510 \mathrm{~nm}$ compared to toluene solution, although at the same time the emergence of new and intense bands peaking at 539, 597, and $660 \mathrm{~nm}$ is observed. These bands can be ascribed to excimer emission, since a spectrum of polycrystalline powder shows only a shift to $495 \mathrm{~nm}$ without changing the shape, except for band broadening (Fig. 7b). This indicates that in MBD thin film, TQPP-Me, molecules have to be arranged face-to-face.

The spectral behavior of spin-coated thin films of TQPP-OC 12 is somewhat different. First of all, the solid state spin-coated and solid solution in polystyrene (PS) TQPP-OC 12 absorption spectra show a red-shift associated with band broadening and a relative intensity change in vibronic transitions (Fig. 8a). Similarly, their fluorescence spectra demonstrate quite different spectral features. The polystyrene solid solution spectrum shows a pronounced vibronic structure compared to its toluene spectrum. Next, the fluorescence spectrum of the raw polycrystalline powder is the mirror image of its absorption spectrum with 


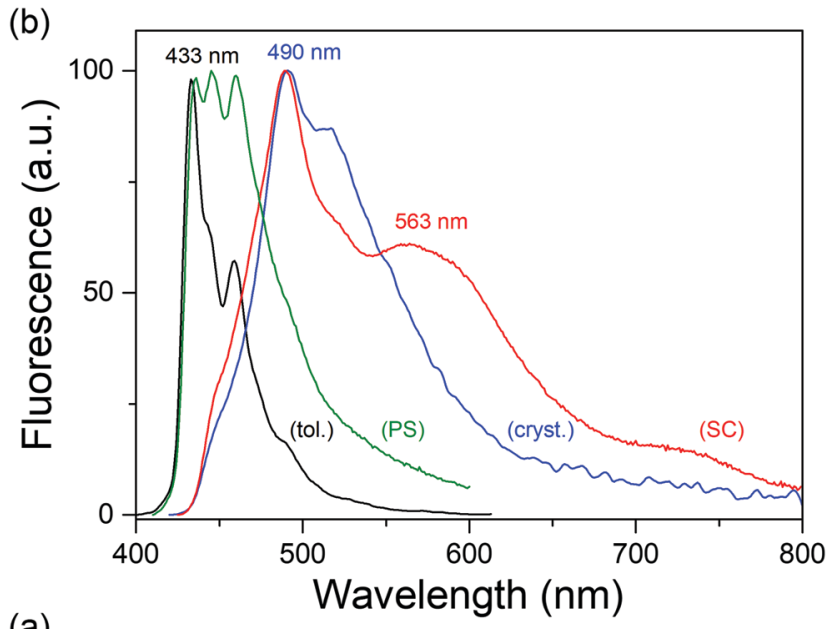

(a)

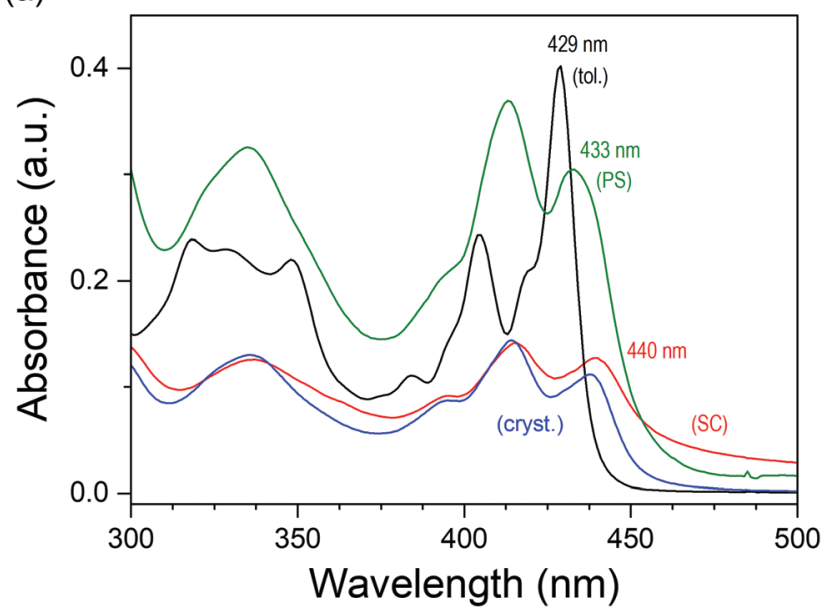

Fig. 8 UV-Vis absorption (a) and fluorescence (b) spectra of TQPP-OC 12 in toluene (tol.) solution, and thin film spin coated (SC) on a quartz substrate. For comparison there are spectra of TQPP-OC 12 in a solid polystyrene matrix (PS), and a polycrystalline layer melted between two quartz plates (cryst.).

the maximum at $490 \mathrm{~nm}$, whereas the spin-coated spectrum shows an additional broad shoulder at $563 \mathrm{~nm}$ and a second less intense one at $715 \mathrm{~nm}$. These changes were developed for the zone-casted sample, which is assumed to be the most aligned. ${ }^{25}$

\section{Spectroscopic ellipsometry study}

Optical properties were further studied using spectroscopic ellipsometry (SE) for the TQPP films. Ellipsometric psi $(\Psi)$ and delta $(\Delta)$ parameters were measured as a function of incidence wavelength $(\lambda)$ over the range of $300-1000 \mathrm{~nm}$ with a resolution of $2 \mathrm{~nm}$, at three incident angles relative to the surface normal from $60^{\circ}$ to $70^{\circ}$ in steps of $5^{\circ} . \Psi(\lambda)$ and $\Delta(\lambda)$ are associated with the Fresnel complex reflection coefficients $r_{\mathrm{p}}$ and $r_{\mathrm{s}}$ for p- and s-polarized light:

$$
\rho \equiv r_{\mathrm{p}} / r_{\mathrm{s}}=\tan (\Psi) \cdot \exp (i \Delta)=f(n, k, d)
$$

and are related to the complex refractive index $(n+i k)$, and thickness $d$ of a layer. Spectroscopic ellipsometry data contain a measured change in the polarization state of light after interaction with a sample. For reflection from an anisotropic sample, the relation between the amplitudes of the reflected $\left(E_{\mathrm{r}}\right)$ and the incident $\left(E_{\mathrm{i}}\right)$ electric field is given by the Jones matrix. ${ }^{45,46}$ The matrix is composed of four reflection coefficients measured for four polarizer and analyzer mutual positions with respect to the plane of incidence. However, samples with uniaxial anisotropy with the symmetry axis perpendicular to the surface reflect the same way regardless of incident polarization and can be acquired using standard isotropic measurement options. ${ }^{47}$

The registered ellipsometric spectrum was analyzed by a model consisting of a stack of three dielectric layers: Si substrate, native silicon oxide of thickness $2 \mathrm{~nm}$ and deposited thin films of TQPP-Me and TQPP-OC 12 . At first, the thickness of the layer was determined in range of the spectrum between 2.05 and $1.24 \mathrm{eV}$ (600-1000 nm) assuming uniaxial anisotropic material - in the plane of the substrate and along the optical axis - which was parameterized by two Cauchy materials. However, the ellipsometric measurements were not very sensitive to the anisotropy, and for both quinoxalinophenanthrophenazine thin films, the isotropic layer was a sufficiently adequate approximation. In principle, the measurements of optical constants from such thin uniaxial films are uncertain. The determined thicknesses were $44 \mathrm{~nm}$ and $75 \mathrm{~nm}$
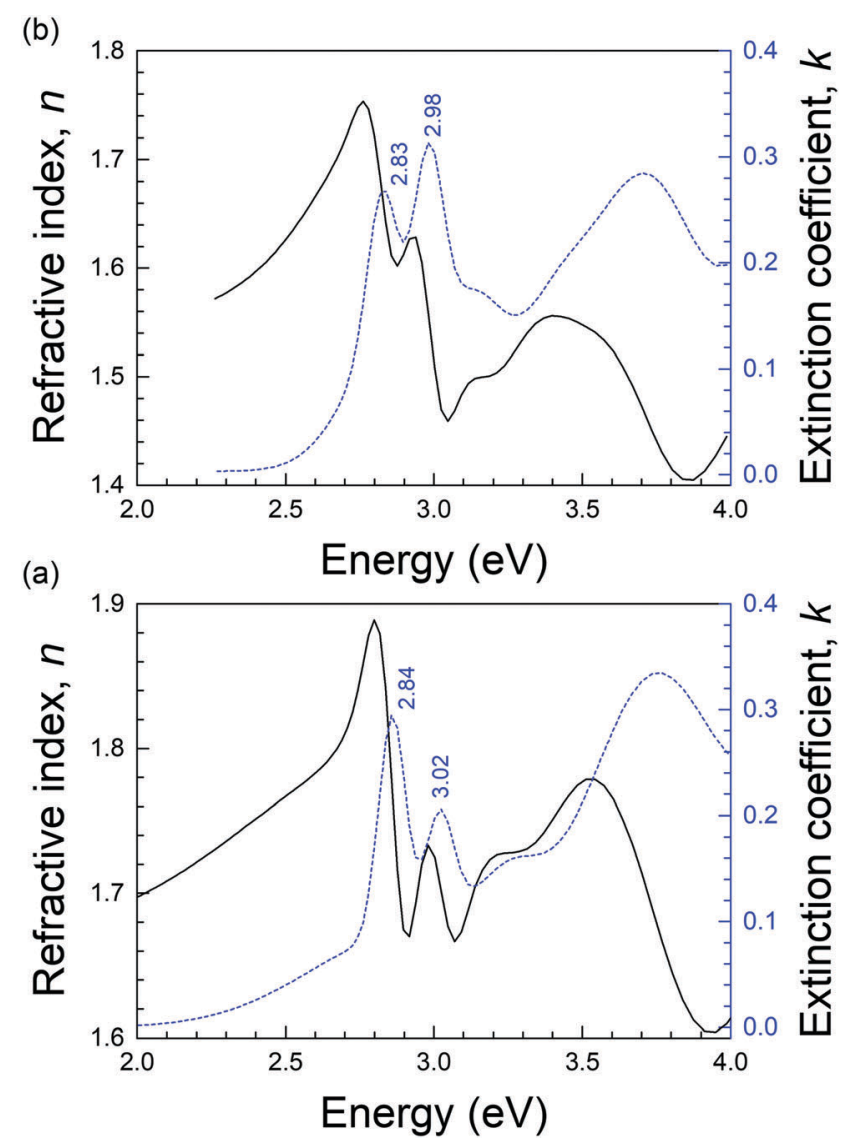

Fig. 9 Refractive index $n$ (solid line), and extinction coefficient $k$ (dashed line) of (a) MBD deposited TQPP-Me layer and (b) spin-coated TQPP-OC 12 layer fitted to an isotropic model layer. 
for TQPP-Me and TQPP-OC 12 , respectively. In the next step, the film thickness was fixed and starting from the low-energy side a point-to-point fit was carried out, resulting in a tabulated set of optical constants, which were then used as reference material for fitting. The experimental data were fitted using a generalized oscillator model of complex dielectric function in the energy range from 1.24 to $4.5 \mathrm{eV}$. For modelling the vibronic transitions Gaussian and harmonic oscillator functions were used and complex refractive index components $n(\lambda)$ and $k(\lambda)$ for thin films of TQPP as a function of energy were determined (Fig. 9). Both extinction coefficients $k$ correspond to their absorption spectra, and $n$ and $k$ dispersions fulfil Kramers-Kronig relations. The maxima of the fitted extinction coefficient were found at $2.87 \mathrm{eV}(432 \mathrm{~nm})$ and $3.0 \mathrm{eV}$ (408 $\mathrm{nm}$ ), for TQPP-Me, and at $2.82 \mathrm{eV}(440 \mathrm{~nm})$ and $2.99 \mathrm{eV}(415 \mathrm{~nm})$ for TQPP-OC 12 .

These ellipsometric results, although satisfactorily designated for isotropic materials (the simplest model), do not exclude uniaxial ordering of the investigated samples. If we accept that the first electronic transition is polarized along the long molecular axis $z$, as was demonstrated in UV-Vis absorption measurements with polarized light, ${ }^{25}$ parallel alignment of the $z$ axis to the substrate will result in an isotropic arrangement of the molecules with respect to the symmetry axis of the sample, and the layered structure will not quite manifest anisotropy in dielectric properties. In principle, the measurements of anisotropy of optical constants from thin uniaxial films with a thickness below $100 \mathrm{~nm}$ may be inherently uncertain.

\section{Conclusions}

We have investigated the structural and optical properties of TQPP-Me and TQPP-OC 12 thin films grown by MBD and spin coating, respectively, on $\left(\mathrm{Si} / \mathrm{SiO}_{2}\right)_{\text {native }}$ and fused silica substrates using polarized GATR infrared spectroscopy, UV-Vis absorption, fluorescence, and AFM microscopy with complementary spectroscopic ellipsometry. For both samples the surface morphology obtained from the AFM measurements consists of crystalline structures with baton-like or packed "dry grass blades" textures which contribute to different degrees of roughness. The differences in morphology are reflected in the investigated properties. The orientation of TQPP-OC ${ }_{12}$ molecules inferred from vibrational modes of polarized GATR indicates that the molecules have the long axis lying parallel and the short axis oriented perpendicular to the $\mathrm{Si} / \mathrm{SiO}_{2}$ substrate. For TQPP-Me both long and short axes are parallel to the substrate surface. In the ellipsometric analysis, a Gaussian and harmonic oscillator model was used to fit the optical parameters of the thin films. However, on this basis the thin layers can be reasonably described as isotropic materials. This finding is not in contradiction with infrared measurements showing that the orientation of the long molecular $z$ axis of TQPP molecules on native silica is close to parallel to the surface.

\section{Conflicts of interest}

There are no conflicts to declare.

\section{Acknowledgements}

This work was supported by the University Research Board (URB) of the American University of Beirut, the Lebanese National Council for Scientific Research (CNRS), and the Kamal A. Shair CRSL. The authors are grateful for this support. The authors would like to thank Dr. D. Batory for his indispensable help with XRD measurements.

\section{References}

1 C. D. Dimitrakopoulos and P. R. Malenfant, Adv. Mater., 2002, 14, 99-117.

2 R. D. Jansen-vanVuuren, A. Armin, A. K. Pandey, P. L. Burn and P. Meredith, Adv. Mater., 2016, 28, 4766-4802.

3 S. A. DiBenedetto, A. Facchetti, M. A. Ratner and T. J. Marks, Adv. Mater., 2009, 21, 1407-1433.

4 J. A. Venables, J. L. Seguin, J. Suzanne and M. Bienfait, Surf. Sci., 1984, 145, 345-363.

5 B. P. G. Rosenfeld and G. Comsa, The Chemical Physics of Solid Surfaces, Elsevier, Amsterdam, 1997.

6 C. Ratsch, A. Zangwill, P. Šmilauer and D. D. Vvedensky, Phys. Rev. Lett., 1994, 72, 3194-3197.

7 S. Kowarik, A. Gerlach and F. Schreiber, J. Phys.: Condens. Matter, 2008, 20, 184005.

8 A. Rao, M. W. B. Wilson, J. M. Hodgkiss, S. Albert-Seifried, H. Bässler and R. H. Friend, J. Am. Chem. Soc., 2010, 132, 12698-12703.

9 R. Eckstein, T. Rödlmeier, T. Glaser, S. Valouch, R. Mauer, U. Lemmer and G. Hernandez-Sosa, Adv. Electron. Mater., 2015, 1, 1500101.

10 G. Pace, A. Grimoldi, D. Natali, M. Sampietro, J. E. Coughlin, G. C. Bazan and M. Caironi, Adv. Mater., 2014, 26, 6773-6777.

11 A. Pierre, I. Deckman, P. B. Lechêne and A. C. Arias, Adv. Mater., 2015, 27, 6411-6417.

12 E. Saracco, B. Bouthinon, J.-M. Verilhac, C. Celle, N. Chevalier, D. Mariolle, O. Dhez and J.-P. Simonato, Adv. Mater., 2013, 25, 6534-6538.

13 D. Baierl, L. Pancheri, M. Schmidt, D. Stoppa, G.-F. Dalla Betta, G. Scarpa and P. Lugli, Nat. Commun., 2012, 3, 1175.

14 A. Tracz, J. K. Jeszka, M. D. Watson, W. Pisula, K. Müllen and T. Pakula, J. Am. Chem. Soc., 2003, 125, 1682-1683.

15 S. E. Fritz, S. M. Martin, C. D. Frisbie, M. D. Ward and M. F. Toney, J. Am. Chem. Soc., 2004, 126, 4084-4085.

16 A. C. Mayer, A. Kazimirov and G. G. Malliaras, Phys. Rev. Lett., 2006, 97, 105503.

17 K. Sakamoto, J. Ueno, K. Bulgarevich and K. Miki, Appl. Phys. Lett., 2012, 100, 123301.

18 C. D. Dimitrakopoulos, A. R. Brown and A. Pomp, J. Appl. Phys., 1996, 80, 2501-2508.

19 M. Shtein, J. Mapel, J. B. Benziger and S. R. Forrest, Appl. Phys. Lett., 2002, 81, 268-270.

20 R. Ruiz, D. Choudhary, B. Nickel, T. Toccoli, K.-C. Chang, A. C. Mayer, P. Clancy, J. M. Blakely, R. L. Headrick, S. Iannotta and G. G. Malliaras, Chem. Mater., 2004, 16, 4497-4508. 
21 F. Schreiber, Phys. Status Solidi A, 2004, 201, 1037-1054.

22 J. W. Lee, K. Kim, J. S. Jung, S. G. Jo, H.-M. Kim, H. S. Lee, J. Kim and J. Joo, Org. Electron., 2012, 13, 2047-2055.

23 S. Le Liepvre, P. Du, D. Kreher, F. Mathevet, A.-J. Attias, C. Fiorini-Debuisschert, L. Douillard and F. Charra, ACS Photonics, 2016, 3, 2291-2296.

24 J. K. Jeszka, J. Ulański and M. Kryszewski, Nature, 1981, 289, 390-391.

25 T. Makowski, R. M. Moustafa, P. Uznanski, W. Zajaczkowski, W. Pisula, A. Tracz and B. R. Kaafarani, J. Phys. Chem. C, 2014, 118, 18736-18745.

26 M. Mas-Torrent, S. Masirek, P. Hadley, N. Crivillers, N. Oxtoby, P. Reuter, J. Veciana, C. Rovira and A. Tracz, Org. Electron., 2008, 9, 143-148.

27 M. Mas-Torrent, P. Hadley, S. T. Bromley, X. Ribas, J. Tarrés, M. Mas, E. Molins, J. Veciana and C. Rovira, J. Am. Chem. Soc., 2004, 126, 8546-8553.

28 C. M. Duffy, J. W. Andreasen, D. W. Breiby, M. M. Nielsen, M. Ando, T. Minakata and H. Sirringhaus, Chem. Mater., 2008, 20, 7252-7259.

29 E. Frạtczak, P. Uznański and M. Moneta, Chem. Phys., 2015, 456, 49-56.

30 L. A. Lucas, D. M. DeLongchamp, L. J. Richter, R. J. Kline, D. A. Fischer, B. R. Kaafarani and G. E. Jabbour, Chem. Mater., 2008, 20, 5743-5749.

31 J. Hu, D. Zhang, S. Jin, S. Z. D. Cheng and F. W. Harris, Chem. Mater., 2004, 16, 4912-4915.

32 S. Leng, B. Wex, L. H. Chan, M. J. Graham, S. Jin, A. J. Jing, K.-U. Jeong, R. M. Van Horn, B. Sun, M. Zhu, B. R. Kaafarani and S. Z. D. Cheng, J. Phys. Chem. B, 2009, 113, 5403-5411. 33 A. Mateo-Alonso, Chem. Soc. Rev., 2014, 43, 6311-6324.
34 B. Wex, A. a. O. El-Ballouli, A. Vanvooren, U. Zschieschang, H. Klauk, J. A. Krause, J. Cornil and B. R. Kaafarani, J. Mol. Struct., 2015, 1093, 144-149.

35 D. Yokoyama, J. Mater. Chem., 2011, 21, 19187-19202.

36 M. Shibata, Y. Sakai and D. Yokoyama, J. Mater. Chem. C, 2015, 3, 11178-11191.

37 S. Yoshiya, S. Maki and Y. Daisuke, Appl. Phys. Express, 2015, 8, 096601.

38 S. Altarawneh, L. Nahar, I. U. Arachchige, A. A. O. El-Ballouli, K. M. Hallal, B. R. Kaafarani, M. G. Rabbani, R. K. Arvapally and H. M. El-Kaderi, J. Mater. Chem. A, 2015, 3, 3006-3010.

39 H. Sirringhaus, P. J. Brown, R. H. Friend, M. M. Nielsen, K. Bechgaard, B. M. W. Langeveld-Voss, A. J. H. Spiering, R. A. J. Janssen, E. W. Meijer, P. Herwig and D. M. de Leeuw, Nature, 1999, 401, 685-688.

40 C. Wang, H. Dong, W. Hu, Y. Liu and D. Zhu, Chem. Rev., 2012, 112, 2208-2267.

41 R. M. Moustafa, J. A. Degheili, D. Patra and B. R. Kaafarani, J. Phys. Chem. A, 2009, 113, 1235-1243.

42 M. Milosevic, S. L. Berets and A. Y. Fadeev, Appl. Spectrosc., 2003, 57, 724-727.

43 J. Michl and E. W. Thulstrup, Spectroscopy with Polarized Light: Solute Alignment by Photoselection, in Liquid Crystals, Polymers, and Membranes, VCH, 1986.

44 J. A. Degheili, R. M. Moustafa, D. Patra and B. R. Kaafarani, J. Phys. Chem. A, 2009, 113, 1244-1249.

45 M. Schubert, Thin Solid Films, 1998, 313, 323-332.

46 M. Schubert, Phys. Rev. B: Condens. Matter Mater. Phys., 1996, 53, 4265-4274.

47 Guide to Using WVASE $^{\mathrm{TM}}$, Spectroscopic Ellipsometry Data Acquisition and Analysis Software, J.A. Woollam Co., Inc., 2012. 